\title{
Hyperparathyroidism in chronic kidney disease: complexities within the commonplace
}

\author{
Michael M Cai, Lawrence P McMahon, Edward R Smith, David S Williams and Stephen G Holt
}

\begin{abstract}
Secondary hyperparathyroidism in patients with chronic kidney disease (CKD) is common and usually caused by associated metabolic abnormalities, in particular, hypocalcaemia and hyperphosphataemia. Nevertheless, other causes of hyperparathyroidism can exist concurrently with CKD, challenging diagnostic interpretation and therapeutic intervention. We present four cases of hyperparathyroidism in patients with CKD that highlight some of these dilemmas.
\end{abstract}

KEY WORDS: hyperparathyroidism, chronic kidney disease, parathyroidectomy, calcimimetic

\section{Key message:}

- Hyperparathyroidism in patients with chronic kidney disease (CKD) is common. While most cases are due to secondary hyperparathyroidism, other causes also need to be considered, especially when the biochemistry is incongruent.

- Imaging is of limited value in distinguishing between secondary and other forms of hyperparathyroidism.

- The use of calcimimetics has altered the therapeutic approach in most instances, but their long-term safety and efficacy, particularly in non-dialysis CKD patients, requires further evaluation.

\section{Introduction}

Secondary hyperparathyroidism (HPT) is common in chronic kidney disease (CKD) as the result of a multitude of metabolic derangements. The prevalence of secondary hyperparathyroidism (sHPT) increases as glomerular filtration rate (GFR) reduces, from $<20 \%$ in patients whose GFR is over $60 \mathrm{ml} / \mathrm{min} / 1.73 \mathrm{~m}^{2}$ to $>50 \%$ in patients with a GFR of less than $30 \mathrm{ml} / \mathrm{min} / 1.73 \mathrm{~m}^{2}{ }^{1}$ The high prevalence of sHPT in CKD, together with the attendant complexities of management, can obscure less frequent causes of hyperparathyroidism, particularly when the presenting clinical and biochemical parameters are similar. We present four cases of

\footnotetext{
Michael M Cai, ${ }^{1}$ renal registrar; Lawrence P McMahon, ${ }^{1}$ director and professor of renal medicine; Edward R Smith, ${ }^{1}$ clinical biochemist; David S Williams, ${ }^{2}$ pathologist; Stephen G Holt, ${ }^{1}$ associate professor of renal medicine

${ }^{1}$ Department of Renal Medicine, Eastern Health Clinical School, Monash University, Melbourne, Australia; ${ }^{2}$ Department of Anatomical Pathology, Austin Health, Melbourne, Australia
}

hyperparathyroidism and renal dysfunction that highlight the diagnostic and therapeutic dilemmas that can arise.

\section{Case 1}

A 66-year-old male with a past history of gastro-oesophageal reflux disease presented to his GP having lost $10 \mathrm{~kg}$ in weight and feeling systemically unwell. Physical examination was unremarkable. Investigations revealed an elevated serum creatinine of $193 \mu \mathrm{mol} / \mathrm{l}$ (this had been recorded at $100 \mu \mathrm{mol} / \mathrm{l}$ two years earlier). The patient was markedly hypercalcaemic (adjusted serum calcium $4.40 \mathrm{mmol} / \mathrm{l}$ ), with a normal serum phosphate $0.71 \mathrm{mmol} / \mathrm{l}$ and an elevated alkaline phosphatase $235 \mathrm{IU} / \mathrm{l}$ (reference range $[R R]$ 30-120). Parathyroid hormone concentration (PTH) was also elevated at $168 \mathrm{pmol} / \mathrm{l}$ (RR 1.0-6.5). Serum and urine electrophoresis and urine microscopy were normal, and there was no evidence of proteinuria or urinary obstruction. The patient was admitted to hospital for intravenous fluids and intravenous bisphosphonate was given to lower his serum calcium. Subsequently, a ${ }^{99} \mathrm{Tc}$-Sestamibi parathyroid scan demonstrated intense uptake in the region of the left lower pole of thyroid, but there was also a focus of less intense uptake in the right lower pole of thyroid, suggesting the possibility of multiple adenomata in association with hyperparathyroidism (Fig 1). A subtotal parathyroidectomy consisting of removal of the right and lower left parathyroid glands was performed, leaving a single gland. Histologically, adenomatous tissue was found in the left lower gland but there was no evidence of adenoma in the other resected glands. The patient's serum calcium returned to normal, but the serum creatinine continued to be elevated at approximately $180 \mu \mathrm{mol} / \mathrm{l}$.

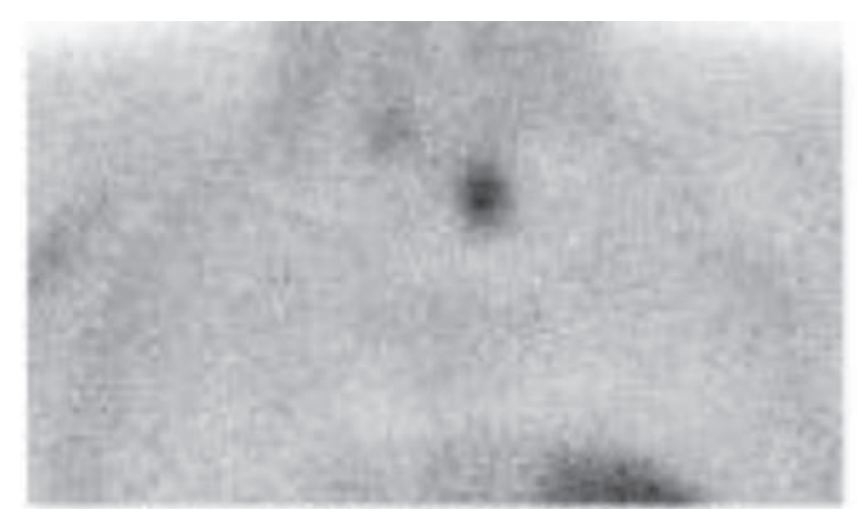

Fig $1 .{ }^{99} \mathrm{Tc}$-Sestamibi scan showing an intense focus of uptake in the region of the left lower pole of the thyroid (Case 1). 
The working diagnosis was primary hyperparathyroidism, with the renal dysfunction probably related to longstanding hypercalcaemia.

\section{Case 2}

A 52-year-old female with no significant family history but with a past history of a congenital single kidney, pseudogout and chondrocalcinosis presented with moderate renal impairment (estimated GFR (eGFR) $36 \mathrm{ml} / \mathrm{min} / 1.73 \mathrm{~m}^{2}$ ), Stage 3 CKD. She was persistently hypercalcaemic, with repeat serum calcium levels measuring between 2.77 and $3.12 \mathrm{mmol} / \mathrm{l}$. Her serum phosphate was in the normal range (between 0.81 and $1.03 \mathrm{mmol} / \mathrm{l}$ ) and plasma PTH was $19.1 \mathrm{pmol} / \mathrm{l}$. A diagnosis of primary hyperparathyroidism was suggested. A ${ }^{99}$ Tc-Sestamibi parathyroid scan demonstrated a single focus of increased
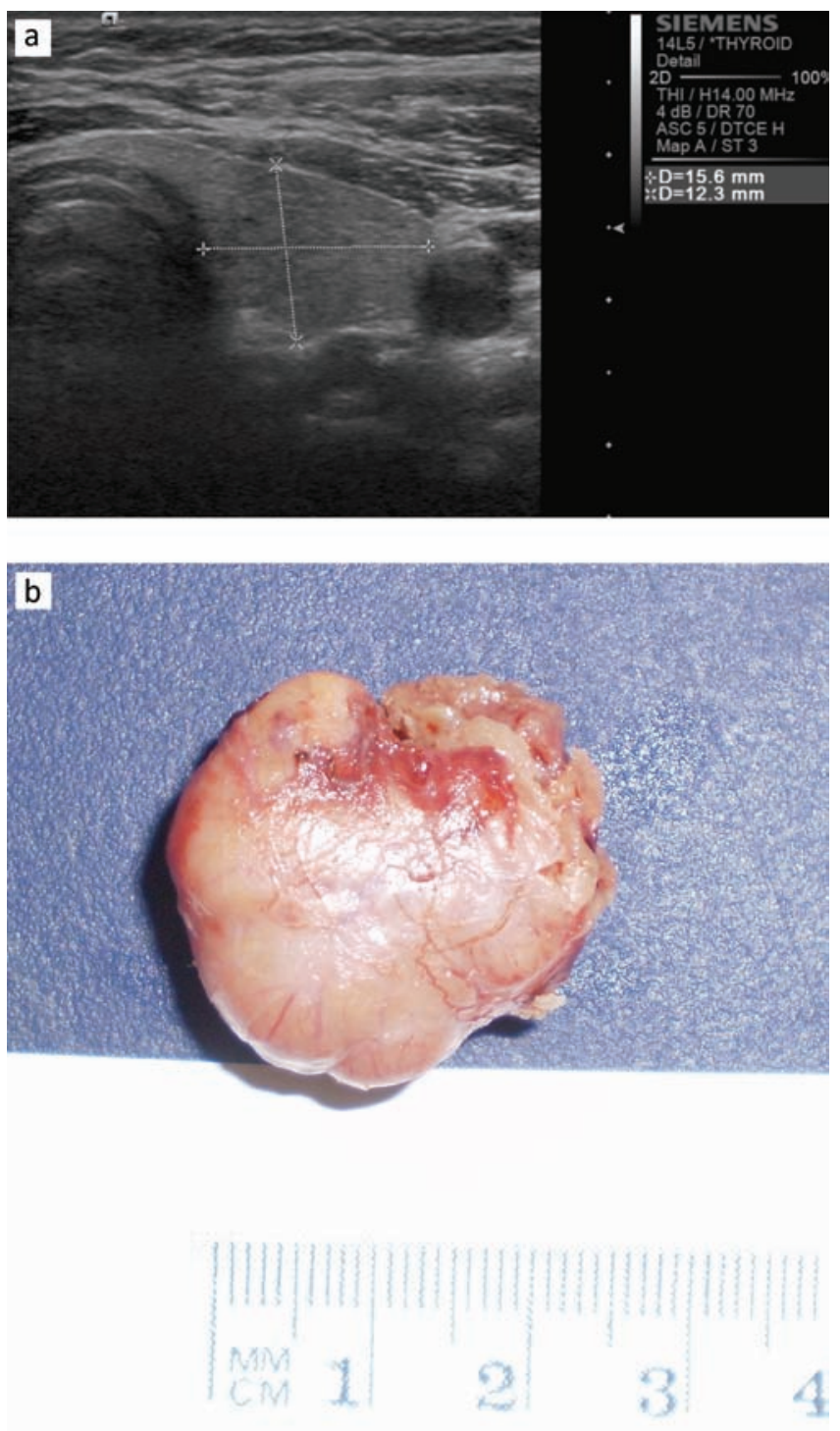

Fig 2 (a) ultrasound showing a very large parathyroid mass. (b) at parathyroidectomy, the mass was easily localized and removed. activity in the lower pole of the right lobe of the thyroid and the patient underwent a single-gland parathyroidectomy. Intraoperatively, the right lower parathyroid gland, thought to be an adenoma, was identified and removed; but histopathological examination failed to identify adenomatous elements. Postoperatively, the patient's serum calcium fell only to the highnormal range (corrected calcium 2.50-2.55 mmol/l) and her PTH remained persistently elevated (10-20 pmol/l). The patient's calcium and plasma PTH concentrations remained stable for a further 10 years, after which her serum calcium climbed again to $2.83 \mathrm{mmol} / \mathrm{l}$, with a serum PTH of $22.7 \mathrm{pmol} / \mathrm{l}$ and a 25-hydroxy vitamin D level of $42 \mathrm{nmol} / \mathrm{l}$. At this time, she was on no diuretic medication. Her concurrent eGFR was $17 \mathrm{ml} / \mathrm{min} / 1.73 \mathrm{~m}^{2}$. A diagnosis of familial hypocalciuric hypercalcaemia $(\mathrm{FHH})$ was considered, and a urine calcium creatinine clearance ratio (UCCR, calculated as urinary calcium/plasma calcium x plasma creatinine/urinary creatinine) was determined. This showed relative hypocalciuria, with a UCCR of 0.005 .

The working diagnosis was secondary hyperparathyroidism, possibly with $\mathrm{FHH}$.

\section{Case 3}

A 25-year-old mother of two presented to her GP with mild fatigue. She described a past history of childhood enuresis, gout and cholelithiasis. There was also a strong family history of end-stage kidney disease. After referral to the renal department, she was considered likely to have an autosomal dominant disorder, uromodulin (UMOD)-associated kidney disease (UAKD), ${ }^{2}$ because a related family member was found to have a mutation in the UMOD gene. Investigation showed a serum creatinine of $120 \mu \mathrm{mol} / \mathrm{l}$, with a reduced creatinine clearance of $54 \mathrm{ml} / \mathrm{min}$, adjusted serum calcium of $2.29 \mathrm{mmol} / \mathrm{l}$ and serum phosphate of $0.51 \mathrm{mmol} / \mathrm{l}$. This patient was lost to renal followup until the age of 33, by which time her renal function had deteriorated and her serum creatinine was $189 \mu \mathrm{mol} / \mathrm{l}$ (eGFR $28 \mathrm{ml} / \mathrm{min} / 1.73 \mathrm{~m}^{2}$ ). Her adjusted serum calcium at that time was $2.37 \mathrm{mmol} / \mathrm{l}$, her serum phosphate $1.1 \mathrm{mmol} / \mathrm{l}$ and her serum uric acid levels also elevated at $0.58 \mathrm{mmol} / \mathrm{l}$. A renal biopsy showed nephrosclerosis with no diagnostic features. The renal dysfunction continued to progress, and four years later, the patient was dialysis-dependent. Before starting dialysis, her PTH was measured at $300 \mathrm{pmol} / \mathrm{l}$ with an adjusted serum calcium of $2.14 \mathrm{mmol} / \mathrm{l}$ and phosphate of $1.77 \mathrm{mmol} / \mathrm{l}$. A ${ }^{99} \mathrm{Tc}-$ Sestamibi parathyroid scan showed a large, right-sided hyperactive gland at the base of the neck, which was also visible on ultrasound scans (as a hypoechoic structure; Fig 2a). The patient underwent a subtotal parathyroidectomy, during which the very large parathyroid gland (9.5 g) was removed (Fig 2b). The contralateral gland was also removed. Histology showed nodular parathyroid hyperplasia in both glands. The PTH measured one day post-operatively had fallen to $11 \mathrm{pmol} / \mathrm{l}$, but within one month it had rebounded to $84 \mathrm{pmol} / \mathrm{l}$. The patient then successfully commenced cinacalcet for medical management of secondary hyperparathyroidism. 
The working diagnosis was secondary hyperparathyroidism resulting from CKD.

\section{Case 4}

A 55-year-old male was found to have hypercalcaemia two weeks after a non-related living-donor kidney transplant. The pretransplant period had been complicated by the unexpected discovery of a donor-specific antibody six days pre-transplant. The antibody was successfully cleared by plasmapheresis and there were no other immediate postoperative complications. The patient's past history included hepatitis B and C infection from IV drug use, end-stage kidney disease resulting from hypertensive nephrosclerosis and secondary hyperparathyroidism requiring calcimimetic treatment (cinacalcet, $60 \mathrm{mg}$ daily). Whilst on dialysis, the patient's adjusted serum calcium concentration ranged between 2.30 and $2.40 \mathrm{mmol} / \mathrm{l}$ and his serum PTH between 20 and $50 \mathrm{pmol} / \mathrm{l}$. Post transplant, the cinacalcet was discontinued and four months after transplantation, the patient's adjusted serum calcium had increased to $2.92 \mathrm{mmol} / \mathrm{l}$. His serum PTH remained elevated (20-30 mmol/l). A ${ }^{99} \mathrm{Tc}$-Sestamibi parathyroid scan demonstrated no focal uptake. A biopsy performed five months post transplantation demonstrated tubulointerstitial calcium deposits with secondary inflammatory changes (Fig 3). The patient was admitted to hospital for intravenous hydration and bisphosphonate therapy, after which his adjusted serum calcium normalised to $2.40 \mathrm{mmol} / \mathrm{l}$. He subsequently recommenced cinacalcet at $30 \mathrm{mg}$ daily, which kept his serum calcium at normal levels.

The working diagnosis was tertiary (autonomous) hyperparathyroidism after renal transplantation.

\section{Discussion}

Secondary HPT as a consequence of CKD is well recognised. It usually presents with a normal or elevated serum phosphate and low or low-normal serum calcium concentration. ${ }^{1}$ Multiple factors mediate the development of hyperparathyroidism in CKD patients. These include hypocalcaemia, decreased 1,25 dihydroxycholecalciferol synthesis and hyperphosphataemia. The differential diagnosis of a raised PTH in the setting of CKD should include primary, secondary and autonomous (tertiary) hyperparathyroidism and, more rarely, $\mathrm{FHH}$ and ectopic PTH secretion.

All causes of hypercalcaemia (including primary HPT (pHPT), myeloma, sarcoidosis, hypervitaminosis D and the milk-alkali syndrome) can result in marked renal dysfunction. Hypercalcaemia damages tubular epithelial cells, particularly those in the loop of Henle and in the collecting and distal tubules, eventually leading to dilatation, atrophy, and fibrosis. ${ }^{3}$ It also down-regulates aquaporin expression which, clinically, can manifest as polyuria, nocturia and reduced urinary concentrating ability. ${ }^{4}$ Hypercalcaemia also has haemodynamic effects, reducing both renal blood flow and glomerular filtration. ${ }^{5}$ Thus, common renal manifestations of pHPT include nephrolithiasis, hypercalciuria and sometimes nephrocalcinosis. ${ }^{6}$

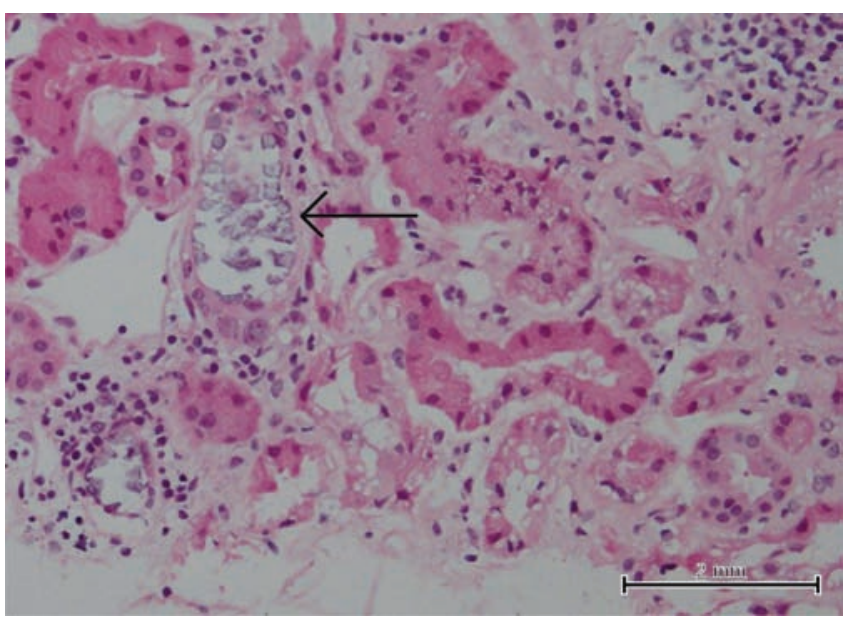

Fig 3. Renal transplant biopsy showing nephrocalcinosis with interstitial calcium deposit (arrow) and inflammatory changes.

Yamashita and co-workers found that hypercalcaemia was more severe in pHPT patients who had renal insufficiency. ${ }^{7}$ Current guidelines suggest that a parathyroidectomy should be considered for most patients with pHPT, specifically those with any of the following: serum calcium concentration $>0.25 \mathrm{mmol} / \mathrm{l}$ above the upper limit of normal, a creatinine clearance of $<60 \mathrm{ml} / \mathrm{min}$, reduced bone density (especially if in the osteoporotic range) and age $<50$ years. ${ }^{8}$ In patients with a high surgical risk, medical management with a calcimimetic agent is usually an effective alternative. ${ }^{9}$

Case 1 illustrates the difficulty often encountered by clinicians in distinguishing pHPT from tertiary HPT (tHPT), as both can present with the triad of renal insufficiency, hypercalcaemia and high PTH. Patients with tHPT usually have moderately severe CKD and a prolonged history of sHPT (ie a period of normo- or hypocalcaemia with concurrent hyperparathyroidism). Serum phosphate is typically elevated in tHPT because of severe renal impairment. By contrast, patients with pHPT typically have a low serum phosphate. The reliance on parathyroid imaging to distinguish between pHPT and tHPT has pitfalls. First, multiglandular involvement occurs in up to $25 \%$ of patients with pHPT. ${ }^{10}$ Second, asymmetric proliferation of the parathyroid glands is common in patients with tHPT and often results in an apparent single focus of intense uptake in imaging studies because of differential tracer retention. ${ }^{10}$ Third, there are other local structures that can take up and retain tracer, giving false positive results. ${ }^{11}$ Thus, multimodal imaging, usually scintigraphy and ultrasound, is recommended.

In Case 2, tHPT was initially considered, but the lack of a prolonged history of CKD and low-normal phosphate on initial presentation argued against this. In addition, PTH was increased only three-fold, whereas in tHPT, elevations are often at least tenfold. The stability of PTH levels for more than decade is also very atypical of tHPT. This evidence leaves pHPT and FHH as possible diagnoses. It is interesting to note that despite her initial partial parathyroidectomy, the patient's post-operative PTH remained elevated and hypercalcaemia eventually recurred. The 
failure of surgery to resolve the condition suggests either multiglandular involvement or the presence of FHH. UCCR is often used as a screening test for $\mathrm{FHH}$, but its validity in CKD patients remains uncertain. Hypocalciuria and 25-hydroxy vitamin D deficiency, both commonly seen in CKD, could potentially increase the false positivity of the test. ${ }^{12,13}$ The patient's serum vitamin D level was slightly reduced, but not deficient, and she was on no thiazide diuretics that may have contributed to a low UCCR. The patient's very low UCCR suggested FHH as a potential co-diagnosis, ${ }^{12}$ but a corroborative family history was lacking.

FHH is a rare, usually autosomal dominant, condition that is associated with defects in the calcium-sensing receptor (CaSR). ${ }^{14}$ The CaSR is expressed in both parathyroid and renal tubular cells, ${ }^{15}$ where loss of function results in increased tubular calcium reabsorption, leading to hypocalciuria that persists even after parathyroidectomy. Analysis of the CaSR gene for clinical use is available in some countries. To diagnose FHH, many experts now suggest using UCCR as a screening test. If positive (UCCR $<0.02$ ), a follow-up CaSR gene analysis would provide a certain diagnosis if a mutation was detected and $98 \%$ sensitivity if no mutations were found. ${ }^{16}$

Case 3 was a severe case of sHPT. The largest gland removed was over 150 times the normal volume. The pre-operative localising ${ }^{99} \mathrm{Tc}$-Sestamibi scan revealed only one hyperfunctioning gland with no other abnormal areas of uptake, suggesting an adenoma. This was consistent with the intraoperative finding of one very large gland, but interestingly, the additional gland that was removed was also enlarged, a finding not detected by scintigraphy.

Discordance between imaging and pathological findings is not uncommon in sHPT; hence there is continuing debate on the value of preoperative imaging, with some authors using it only for recurrent or persistent disease. ${ }^{17}$ Indeed, the choice of modality that is best for imaging parathyroid glands is also contentious. Imaging of the normal parathyroid gland is difficult as this tissue is not easily distinguished from surrounding tissue. As the gland expands and increases in cellularity, however, it becomes increasingly easy to detect by ultrasound. Highresolution colour Doppler ultrasound now also enables more accurate assessment of changes in parathyroid volume. ${ }^{18}$ ${ }^{99} \mathrm{Tc}$-Sestamibi imaging helps to localise parathyroid tissue but provides little anatomical localisation and often must be used in combination with another imaging modality. ${ }^{19}$ Thyroid uptake of radiotracers can also be a source of error and some authors advocate a subtraction scan by imaging with ${ }^{123} \mathrm{I}$ or ${ }^{99} \mathrm{Tc}$-Pertechnetate. Previous studies have demonstrated the poor sensitivity of various imaging techniques in identifying all affected glands. ${ }^{17}$ Some authors advocate a combination of ${ }^{99} \mathrm{Tc}$-Sestamibi single photon emission computed tomography (SPECT) with CT to gain high sensitivity and accuracy gland localisation. ${ }^{19}$ MRI can also be helpful, especially when combined with scintigraphy. ${ }^{20}$

Another noteworthy lesson from Case 3 was the fact that severe secondary hyperparathyroidism can occur early in CKD: recent guidelines suggest that PTH concentrations should be monitored when the GFR falls below $60 \mathrm{ml} / \mathrm{min} / 1.73 \mathrm{~m}^{2}{ }^{21}$ The ideal target for PTH is not known, but the current Kidney Disease Outcome Quality Initiative (KDOQI) guidelines suggests that the PTH should be kept at between 3.85 and $7.7 \mathrm{pmol} / \mathrm{l}$ in Stage 3 CKD, and between 7.7 and $12.1 \mathrm{pmol} / \mathrm{l}$ in Stage 4 $\mathrm{CKD} .{ }^{22}$ The interpretation of PTH concentration is further complicated by the high intra-individual biological variation in PTH secretion in CKD patients; a single measurement rarely provides an accurate picture of parathyroid activity. ${ }^{23}$ Indeed, PTH itself might not be the best molecule to measure in early CKD. The hormone fibroblast growth factor-23 (FGF-23) could be a more appropriate measure when assessing bone and mineral disorders. FGF-23 is a phosphaturic hormone that down-regulates the activation of vitamin D in the kidney. This hormone is elevated long before changes are seen in calcium, phosphate or $\mathrm{PTH},{ }^{24}$ but because of its instability in plasma samples, it is not easy to measure. ${ }^{25}$

In the past decade, parathyroidectomy in dialysis patients has been substantially delayed by the introduction of calcimimetic agents. Parathyroidectomy continues to be performed, but at a markedly decreased frequency. The role of calcimimetics in nondialysis patients is less well defined. In randomised, controlled, placebo-controlled trials, cinacalcet treatment reduced PTH levels, but it did so at the cost of significant hypocalcaemia and hyperphosphataemia. ${ }^{26}$ Thus, early routine use of calcimimetics in CKD is currently neither recommended nor licensed. Nevertheless, there may be individual cases in which pre-dialysis use might help to prevent severe secondary hyperparathyroidism.

The last case presents a common scenario of hypercalcaemia post renal transplantation. Secondary HPT occurs frequently in CKD patients and, although pHPT cannot be excluded completely without histology, the symmetrical uptake and excretion of tracer in the parathyroid scan in this case argues against this. Although renal transplantation eventually corrects the sHPT for many, the correction can take time and is incomplete in $50 \%$ of recipients. ${ }^{27}$ As demonstrated in this patient, marked hypercalcaemia can cause graft dysfunction and nephrocalcinosis. Traditionally, hyperparathyroidism post transplantation has been managed with phosphate supplementation, bisphosphonates and, in refractory cases, parathyroidectomy. In some cases, this procedure has been shown to be deleterious to graft function. ${ }^{28}$ Numerous case series have now reported the effectiveness of cinacalcet in reducing serum PTH and calcium after transplantation (see review by Copley ${ }^{29}$ ). There are, however, at least three case reports of graft dysfunction resulting from nephrocalcinosis after the initiation of cinacalcet treatment. ${ }^{29-32} \mathrm{CaSR}$ is present in both parathyroid tissues and renal tubules. ${ }^{33}$ In addition to the suppression of PTH secretion, cinacalcet can also interact directly with the CaSR expressed in renal tubules, resulting in increased calcium excretion that is independent of PTH's action on renal tubules. ${ }^{14}$ Therefore, cinacalcet might exert hypercalciuric effects through both $\mathrm{PTH}$-dependent and -independent mechanisms. With these concerns in mind, the results of a randomised placebo-controlled trial (see clinicaltrials.gov NCT 00975000, due for completion in 2013) will 
hopefully provide a more definitive answer on the efficacy and safety of cinacalcet in this setting.

In conclusion, renal dysfunction and hyperparathyroidism often co-exist. Although usually caused by secondary hyperparathyroidism, incongruent biochemistry can challenge both the diagnosis and treatment of these conditions.

\section{References}

1. Levin A, Bakris GL, Molitch M et al. Prevalence of abnormal serum vitamin D, PTH, calcium, and phosphorus in patients with chronic kidney disease: results of the study to evaluate early kidney disease. Kidney Int 2006;71:31-8.

2. Rampoldi L, Sclori F, Amoroso A et al. The rediscovery of uromodulin (Tamm-Horsfall protein): from tubulointerstitial nephropathy to chronic kidney disease. Kidney Int 2011;80:338-47.

3. Carone FA, Epstein FH, Beck D, Levitin H. The effects upon the kidney of transient hypercalcemia induced by parathyroid extract. Am J Pathol 1960;36:77-103.

4. Procino G, Carmosino M, Tamma G et al. Extracellular calcium antagonizes forskolin-induced aquaporin 2 trafficking in collecting duct cells. Kidney Int 2004;66:2245-55.

5. Levi M, Ellis MA, Berl T. Control of renal hemodynamics and glomerular filtration rate in chronic hypercalcemia. J Clin Invest 1983;71:1624-32.

6. Silverberg SJ, Shane E, Jacobs TP et al. A 10-year prospective study of primary hyperparathyroidism with or without parathyroid surgery. $N$ Engl J Med 1999;341:1249-55.

7. Yamashita H, Noguchi S, Uchino S et al. Influence of renal function on clinico-pathological features of primary hyperparathyroidism. Eur J Endocrinol 2003;148:597-602.

8. Bilezikian JP, Khan AA, Potts JT. Guidelines for the management of asymptomatic primary hyperparathyroidism: Summary Statement from the Third International Workshop. J Clin Endocrinol Metab 2009;94:335-9.

9. Peacock M, Bilezikian JP, Bolognese MA et al. Cinacalcet $\mathrm{HCl}$ reduces hypercalcemia in primary hyperparathyroidism across a wide spectrum of disease severity. J Clin Endocrinol Metab 2011;96:e9-18.

10. Kebebew E, Hwang J, Reiff E et al. Predictors of single-gland vs multigland parathyroid disease in primary hyperparathyroidism: a simple and accurate scoring model. Arch Surg 2006;141:777-82.

11. Johnson NA, Carty SE, Tublin ME. Parathyroid imaging. Radiol Clin North Am 2011;49:489-509.

12. Jayasena CN, Mahmud M, Palazzo F et al. Utility of urine calcium-tocreatinine ratio to diagnose primary hyperparathyroidism in asymptomatic hypercalcaemic patients with vitamin D deficiency. Ann Clin Biochem 2011;48:126-9.

13. Spiegel DM, Brady K. Calcium balance in normal individuals and in patients with chronic kidney disease on low and high-calcium diets. Kidney Int 2012; 81:1116-22.

14. Pollak MR, Brown EM, Chou YWH et al. Mutations in the human $\mathrm{Ca}^{2+}$-sensing receptor gene cause familial hypocalciuric hypercalcemia and neonatal severe hyperparathyroidism. Cell 1993;75:1297303.

15. Vargas-Poussou R, Huang C, Huilin P et al. Functional characterization of a calcium-sensing receptor mutation in severe autosomal dominant hypocalcemia with a Bartter-like syndrome. J Am Soc Nephrol 2002;13:2259-66.
16. Christensen SE, Nissen PH, Vestergaard P, Mosekilde L. Familial hypocalciuric hypercalcaemia: a review. Curr Opin Endocrinol Diabetes Obes 2011;18:359-70.

17. Lai ECH, Ching ASC, Leong HT. Secondary and tertiary hyperparathyroidism: role of preoperative localization. ANZ J Surg 2007;77: 880-2.

18. Meola M, Petrucci I, Colombini E, Barsotti G. Use of ultrasound to assess the response to therapy for secondary hyperparathyroidism. Am J Kidney Dis 2011;58:485-91.

19. Lumachi F, Ermani M, Basso S et al. Localization of parathyroid tumours in the minimally invasive era: which technique should be chosen? Population-based analysis of 253 patients undergoing parathyroidectomy and factors affecting parathyroid gland detection. Endocr Relat Cancer 2001;8:63-9.

20. Numerow LM, Morita ET, Clark OH, Higgins CB. Hyperparathyroidism: a comparison of sestimibi scintigraphy, MRI, and ultrasonography. J Magn Reson Imaging 1995;5:702-8.

21. Anon. Diagnosis of CKD-MBD: biochemical abnormalities. Kidney Int 2009;76:S22-49.

22. Anon. Treatment of CKD-MBD targeted at lowering high serum phosphorus and maintaining serum calcium. Kidney Int 2009;76:S5099.

23. Gardham C, Steves PE, Delaney MP et al. Variability of parathyroid hormone and other markers of bone mineral metabolism in patients receiving hemodialysis. Clin J Am Soc Nephrol 2010;5:1261-7.

24. Wolf M. Fibroblast growth factor 23 and the future of phosphorus management. Curr Opin Nephrol Hypertens, 2009;18:463-8.

25. Smith ER, Ford ML, Tomlinson LA et al. Instability of fibroblast growth factor-23 (FGF-23): implications for clinical studies. Clinica Chimica Acta 2011;412:1008-11.

26. Chonchol M, Locatelli F, Abboud HE et al. A randomized, doubleblind, placebo-controlled study to assess the efficacy and safety of cinacalcet $\mathrm{HCl}$ in participants with $\mathrm{CKD}$ not receiving dialysis. Am J Kidney Dis 2009;53:197-207.

27. Reinhardt W, Bartelworth H, Jockenhovel F et al. Sequential changes of biochemical bone parameters after kidney transplantation. Nephrol Dial Transplant 1998;13:436-42.

28. Lewin E, Olgaard K. Parathyroidectomy vs calcimimetics for treatment of persistent hyperparathyroidism after kidney transplantation. Nephrol Dial Transplant 2006;21:1766-9.

29. Copley JB, Wuthrich RP. Therapeutic management of post-kidney transplant hyperparathyroidism. Clin Transplant 2011;25:24-39.

30. Seikrit C, Nuhlfeld A, Groene HJ, Floege J. Renal allograft failure in a hyperparathyroid patient following initiation of a calcimemetic. Nat Rev Nephrol 2011;7:237-41.

31. Peng LW, Logan JL, James SH et al. Cinacalcet-associated graft dysfunction and nephrocalcinosis in a kidney transplant recipient. Am J Med 2007;120:e7-9.

32. Esposito L, Rostaing L, Gennero I et al. Hypercalciuria induced by a high dose of cinacalcet in a renal-transplant recipient. Clin Nephrol 2007;68:245-8.

33. Herbert SC. Extracellular calcium-sensing receptor: implications for calcium and magnesium handling in the kidney. Kidney Int $1996 ; 50: 2129-39$.

Address for correspondence: Dr Michael Cai, Department of Renal Medicine, Eastern Health Clinical School, Monash University, Level 2, 5 Arnold Street, Box Hill, Victoria 3128, Australia.

Email: Michael.Cai@easternhealth.org.au 